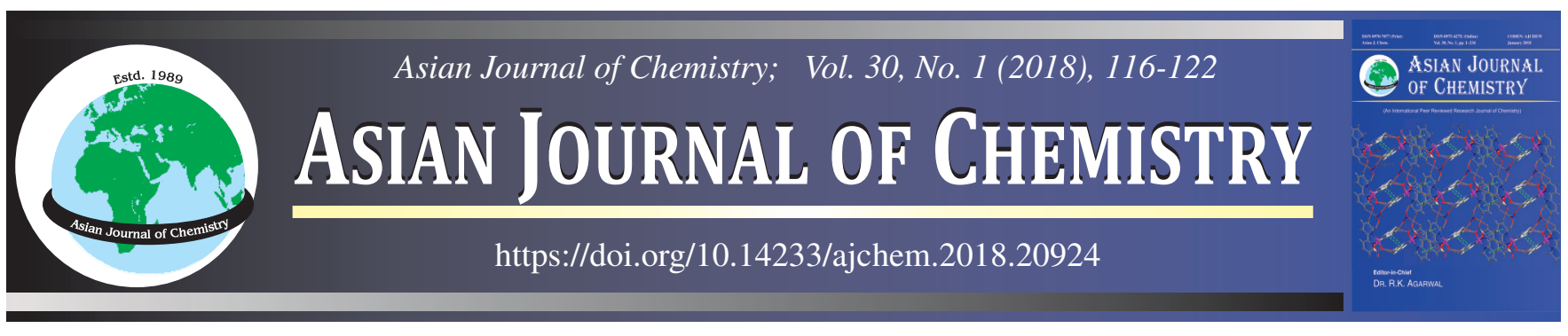

\title{
UV Protection and Antibacterial Treatment of Wool using Green Silver Nanoparticles
}

\author{
Sakil Mahmud ${ }^{1,2, *}$, Md. Nahid Pervez ${ }^{1,3}$, Md. Ahsan Habid ${ }^{1,4}$, Mst. Zakia Sultana $^{1,5}$ and Hui-Hong Liu ${ }^{1, *}$
}

${ }^{1}$ School of Chemistry and Chemical Engineering, Wuhan Textile University, Wuhan 430200, P.R. China

${ }^{2}$ Ningbo Institute of Material Technology and Engineering, Chinese Academy of Sciences, Ningbo 315201, P.R. China

${ }^{3}$ Research Institute of Flexible Materials, School of Textiles \& Design, Heriot-Watt University, TD1 3HF, UK

${ }^{4}$ Institutes of Chemistry, Chinese Academy of Sciences, Beijing 100190, P.R. China

${ }^{5}$ NUS Graduate School for Integrative Science \& Engineering, National University of Singapore, 28 Medical Drive, Singapore 117456, Singapore

*Corresponding authors: E-mail: sakilhabib@gmail.com; huihongliu@wtu.edu.cn

Herein, we attempted a new green based silver nanoparticles employing sodium alginate (Na-Alg) as reducing agent to functionalize wool fabric surface. This research aims to enhance the antimicrobial activity and UV resistance of wool fabrics treated with synthesized silver nanoparticles. The resulting products were characterized by scanning electron microscopy, Fourier transform infrared spectroscopy spectra (FTIR), X-ray diffraction (XRD), thermogravimetric analysis (TGA) and UV-visible absorbance. The results specified that silver nanoparticles (AgNPs) were successfully assembled on wool surface when liquor $\mathrm{pH}$ and temperature of application medium were adjusted to 4 and $40^{\circ} \mathrm{C}$, respectively for $2 \mathrm{~h}$. Thermogravimetric analysis indicated that the combined finishing of wool fabric with AgNPs could improve its thermal property. It was observed that the presence of green based AgNPs significantly enhanced the UV resistance and antibacterial activity of fabrics particularly for samples coated with $70 \mathrm{ppm}$ AgNPs. These promising results support that the present approach is useful for the fabrication of antimicrobial and UV protector fabrics.

Keywords: Green synthesis, Silver nanoparticles, Wool, UV Resistance, Antibacterial activity.

\section{INTRODUCTION}

It is well documented that due to providing a sufficient energy source of nutrients natural fibers are considered to be viable route for culturing different microorganism and among all natural protein fibers wool has been demonstrated as a superior material for winter and medical textiles due to its resilience and comfort properties [1]. The additional sophisticated properties of wool have been claim urgency since people's improving their living standard and it is recommended that can be achieved by acquiring multi-functional performances such as preference of durable antibacterial activity and ultraviolet protection for the sake of health considerations [2,3]. Traditionally, in case of industrial application some antimicrobial agents have been introduced including quaternary ammonium salts; metal salts solutions and antibiotics but the existence of toxicity and less effective profile is not allowed them to use in filters, textiles, health foods and for the exclusions of pollution. Taking into considerations about economic and ecological issues in the modern coating industries and support to prevent harmful pathogens and detrimental UV-radiations, employment of nanoparticles could be an obvious choice [4]. Recently, scientific attention has been withdrawn to modify surface of cellulose and protein fibers by incorporating metal and metal assisted nanoparticles (NPs) due to perform some multifunctional attributes such as antibacterial, UV protection, antistatic and self-cleaning [5,6]. Among the metallic nanoparticles, AgNP has nanosize between $1 \mathrm{~nm}$ and $100 \mathrm{~nm}$ and consider one of the best candidates for several applications including biosensing, microbial activities, drug delivery, catalysis, electrochemical, conductivity, etc. [7,8], with exponentially accretion production. Generally, a number of physical and chemical methods are available in order to preparing the metallic nanoparticles $[9,10]$, which are not environment friendly. Ideally, green chemistry perspective synthesis of nanoparticles associated with three main tones is the choice of the solvent medium, environmentally benign reducing agent, and source of non-toxic material for the stabilization of the nanoparticles [11]. Some researchers have reported green preparation of nanoparticles through natural polymers like chitosan, soluble starch, polypeptide, heparin and hyaluronan used as reducing and stabilizing agent [12].

Recently, the polysaccharide based materials has been associated with the synthesis of green AgNPs through an ecofriendly benign solvent i.e., water and polysaccharides as 
capping agents [13]. Based on this approach, we have produced the green-chemistry type AgNP synthesis processes in this research. The wool fiber is concentrated of keratin protein, cuticle cell is the part of outermost region and surface of it (a fatty layer of 18-methyl eicosanoic acid) mainly covalently bounded with cuticle cell through thioester linkage and nature of wool surface is hydrophobic because of this structural composition [14]. Moreover, this promising property of wool fiber helps them to be an ideal for binding with metal ions and act as a suitable platform to culture of metal nanoparticles with polyfunctional ligands [15-18].

The present research aims to devise a sustainable route for the green synthesized AgNPs by using Na-Alg as a reducing and stabilizing agent coating on wool fabric with durable UV protection and antibacterial property. As synthesized AgNPs were applied on fabric by exhaustion method which comprises of three steps i.e., initially wool sample was scoured well than impregnated with freshly prepared AgNPs colloids under optimized condition and finally rinsed out to remove unfix nanoparticles.

\section{EXPERIMENTAL}

Wool fabrics were obtained from Jiangsu Shenzhou Woolen Co., Ltd. China. Materials used to synthesize AgNPs were $\mathrm{AgNO}_{3}$ (Shanghai Zhanyun Chemical Co., Ltd. Shanghai, China), Na-Alg (Qingdao Yingfei Chemical Co., Ltd. Qingdao, China) and $\mathrm{NaOH}$ (Sinopharm Chemical Reagent Co., Ltd., Suzhou, China). Non-ionic commercial detergents were used to wash the wool sample. Deionized water was used throughout the experiment.

Synthesis of AgNPs: The green synthesis of AgNPs was accomplished using an indigenous protocol made in our laboratory. In brief, $1 \mathrm{~mL}$ of $\mathrm{Na}-\mathrm{Alg}(1 \% \mathrm{w} / \mathrm{v})$ solution was mixed in $4 \mathrm{~mL}$ of silver nitrate $(0.001 \mathrm{M})$ solution with addition of $\mathrm{NaOH}$ solution $(1 \mathrm{~mL}$ of $0.01 \mathrm{M})$ to maintain $\mathrm{pH} 11$. This solution was stirred for $1 \mathrm{~min}$ and heated in the water bath $\left(60^{\circ} \mathrm{C}\right)$ for $40 \mathrm{~min}$. The transparent colourless solution was converted to pale yellow and then into brownish-red colour indicated the formation of AgNPs. At this end, the prepared solution was evaluated by using UV-visible spectra to affirm the production of AgNPs.

Wool functionalization: The wool fabrics were washed in a bath containing $2 \mathrm{~g} \mathrm{~L}^{-1}$ non-ionic detergent with $\mathrm{L}: \mathrm{M}=$ 50:1 (liquor to material ratio) at $50^{\circ} \mathrm{C}$ for $15 \mathrm{~min}$. Then, those were rinsed with distilled water and dried at $110^{\circ} \mathrm{C}$ for $5 \mathrm{~min}$. Silver nanoparticles were applied on wool surface by using exhaustion methods. The $\mathrm{pH}$ value of AgNPs solution was adjusted to 4 with acetic acid and then the scoured wool fabric samples were immersed in an aqueous solution containing AgNPs (liquor ratio of $90: 1$ ) at $45^{\circ} \mathrm{C}$ for $2 \mathrm{~h}$ (hereafter referred as optimized condition) with different concentrations of AgNPs. Afterward, the treated wool samples were rinsed with running deionized water.

The UV-visible spectra of wool fabrics with AgNPs were recorded using a spectrophotometer (Shimadzu Corporation, Kyoto, Japan). The morphologies of samples were examined using a scanning electron microscope (SEM) (JEOL, Tokyo, Japan) after gold coating. During SEM test, an EDS spectrum was collected to analyze the chemical elements of the samples. Transmission electron microscopy (TEM) (Hitachi H-7600, Tokyo, Japan) was used to study the particle size distribution of AgNPs. The crystal behaviour of samples was obtained using an X-ray diffractometer (Bruker D8 ADVANCE, Karlsruhe, Germany). FTIR measurements were performed with a Bruker Tensor 27 (Bruker Corporation, Tensor 27, Karlsruhe, Germany) in a normal transmission mode. Thermal behaviour of samples was performed on a thermogravimetric analyzer (Mettler-Toledo Corp., Switzerland) at a heating rate of $10^{\circ} \mathrm{C} / \mathrm{min}$ under nitrogen atmosphere. UV protective characteristics of original and silver coated fabrics were determined in accordance with Australian/ New Zealand Standard AS/NZS 4399:1996 by using UV-visible spectrophotometer. Antibacterial efficiency of green synthesized AgNPs in wool fabrics was determined against Staphylococcus aureus ATCC 6538 as a Gram-positive and Escherichia coli ATCC 8739 as Gram-negative bacteria [18]. These bacteria were cultured in nutrient agar medium (cooled to $47 \pm 2{ }^{\circ} \mathrm{C}$ ) was dispensed into each petri dish by pouring $15 \pm 2 \mathrm{~mL}$ and used as inocula, where circular wool fabrics were placed uniformly. After incubation at $37^{\circ} \mathrm{C}$ for $12 \mathrm{~h}$, the pictures of the agar plates were captured and antimicrobial effects of the samples were assessed based on the area of bacterial inhibition zones. Wool fabrics were tested for the tensile properties by using Instron 3365 universal testing machine (Instron Corporation, Boston, MA, USA). before and after AgNPs treatment. The crease recovery angle of the samples was determined as perAATCC Test method 66-2003 using a crease recovery tester (SASMIRA, Mumbai, India). The stiffness in terms of bending length of untreated and AgNPs treated sample was tested as per AATCC Test method 115-2005 using profile stiffness tester (SASMIRA, Mumbai, India).

\section{RESULTS AND DISCUSSION}

In the present work, AgNPs had been synthesized in a 'green' method using natural biopolymer $\mathrm{Na}-\mathrm{Alg}$ with the aid of certainly heating an aqueous combination of $\mathrm{Na}-\mathrm{Alg}$ and $\mathrm{AgNO}_{3}$. The preliminary affirmation of nanoparticles formation turned into ascertained via recording the absorbance of colloidal suspension using UV-visible spectrophotometry in the range of 200-700 $\mathrm{nm}$ and colour change observation. The solution colour turned into reddish brown indicated the reduction of silver ions and appropriate the acknowledged synthesis of AgNPs is apparent (Fig. 1A inset). The distinctive colours of AgNPs are due to a phenomenon known as plasmon absorbance. Incident light creates oscillations in conduction electrons on the surface of nanoparticles and electromagnetic radiation is enamored [19]. Fig. 1A shows the UV-vis spectra of pure silver nitrate, Na-Alg and AgNPs. In Na-Alg, the band at $301 \mathrm{~nm}$ exhibited by $\mathrm{AgNO}_{3}$ in aqueous solution disappeared indicating the possible chelation of $\mathrm{Ag}^{+}$by $\mathrm{OH}$ and $\mathrm{COOH}$ groups of alginate. The $\mathrm{Ag}^{+}$chelate produces $\mathrm{Ag}^{0}$ on heating. The intensity and role of the shoulder of alginate spectrum at about $290 \mathrm{~nm}$ turned barely shifted, confirming that there have been interactions among the polymer and metallic precursor. The UVvisible spectrum of AgNPs showed a characteristic peak at $400 \mathrm{~nm}$ [20]. For the synthesis of AgNPs, the typically accepted mechanism shows a two-step process, i.e. atom formation 
follwed by the polymerization of atoms. In the first step, a portion of metal ions in solution is reduced by reducing groups. The reasons is due to fact that atoms produced act as nucleation centers and catalyze the metal ions in bulk solution. Compared with other water-soluble polymers, $\mathrm{Na}-\mathrm{Alg}$ is an anionic polymer with high charge density. The negatively charged alginate allows the appeal of positively charged silver ions to the polymeric chains, which have been then decreased by way of present reducing corporations. The resulting surface negative charge of alginate fragments containing carboxylic groups stabilizes the nanoparticles against coalescing due to electrostatic repulsion and steric outcomes [21].

Fig. 1B shows pure Na-Alg spectra which represent the two primary peaks at about $3435 \mathrm{~cm}^{-1}$ (stretching of $\mathrm{OH}$ groups) and at $2025 \mathrm{~cm}^{-1}$ (CH stretching of $\mathrm{CH}_{2}$ groups). The $\mathrm{CO}$ asymmetrical stretching at $1609 \mathrm{~cm}^{-1}$ along with a weaker symmetrical stretching band at $1414 \mathrm{~cm}^{-1}$ [22] were empiric due to salt nature of carboxylic acid groups at pure Na-Alg. An accelerated band at $1083 \mathrm{~cm}^{-1}$ is apery the stretching of C-O-C group; the peaks at $601 \mathrm{~cm}^{-1}$ belong to $\mathrm{C}-\mathrm{O}-\mathrm{C}$ glycosidic linkage (ring breathing) [23]. They are accompanying to its saccharide structure [24]. In case of the AgNPs, the band of $\mathrm{CO}_{2}{ }^{-}$is shifted to $1613 \mathrm{~cm}^{-1}$ in addition the peaks of 3435 and $1414 \mathrm{~cm}^{-1}$ shift to 3422 and $1384 \mathrm{~cm}^{-1}$ respectively due to ring stretching of metal groups and indicating that $\mathrm{Na}-\mathrm{Alg}$ was doped by $\mathrm{NO}_{3}{ }^{-}$ because of stabilization of AgNPs. The comparison of FTIR spectra between Na-Alg and $\mathrm{Na}-\mathrm{Alg} / \mathrm{AgNPs}$ showed only minor changes in the position as well as the absorption bands. Thereby, FT-IR spectrum confirms that AgNPs have been capped with the aid of the lone pair electrons around the oxygen atoms in organic compound in $\mathrm{Na}-\mathrm{Alg}$ with van der Waals interaction forces [25]. Fig. 1C presents SEM image of Na-Alg loaded with AgNPs. It is manifested that AgNPs synthesized by greenly can cautiously be admired as nanoparticles with spherical nature. The agglomeration of AgNPs was due to interactions of hydrogen bond and electrostatic interactions between the bioorganic capping molecules apprenticed to the AgNPs [26]. TEM image with corresponding particle size distribution (PSD) of as-prepared AgNPs is shown in Fig. 1D. From the image, it could be seen that the AgNPs located are round in form and nicely separated in aqueous medium which are covered via layer. The layer can be the phytoconstituents of Na-Alg. In addition, the aggregation is lower because of less collision of AgNPs. From the scale distribution picture, it could be ascribed that the maximum number of nanoparticles are inside the length among 6 to $10 \mathrm{~nm}$. The reductive properties of Na-Alg are notably more desirable owing to base hydrolysis with the formation of low molecular weight reducing fragments and consequently, reflecting the twin function of Na-Alg as stabilizing and reducing agent in alkaline medium [27]. The additional support of reduction of $\mathrm{Ag}^{+}$ions to elemental silver was confirmed by EDS analysis (Fig. 1E). The optical absorption peak is observed at $3 \mathrm{keV}$, which is typical for the absorption of metallic silver nanocrystalline due to surface plasmon resonance [28], which confirms the presence of nanocrystalline elemental silver. Fig. 1F suggested the typical XRD pattern of AgNPs prepared with several distinct diffraction peaks at approximately $38.1^{\circ}, 44.2^{\circ}, 64.3^{\circ}$ and $77.4^{\circ}$ are assigned to reflections from the (111), (200), (220) and (311) planes of the silver crystal, respectively, which confirms the existence of silver and further on the basis that they can be indexed as face-centered-cubic (FCC) structure of silver. These peaks are
(A)

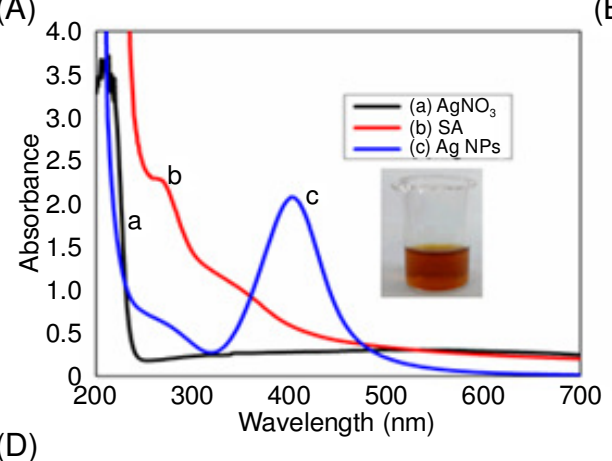

(D)

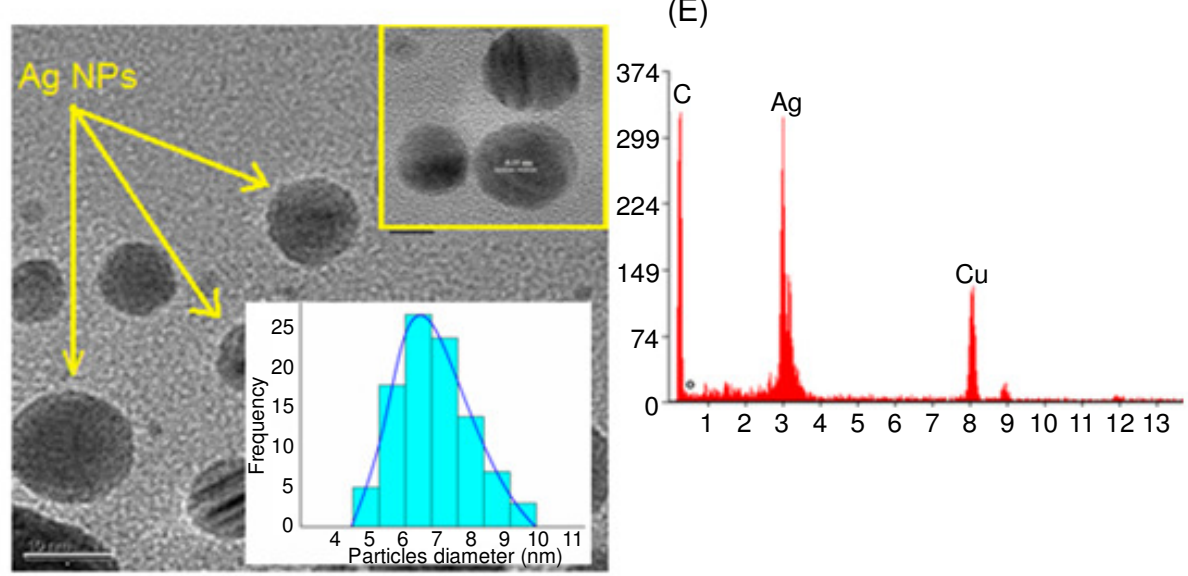

(B)

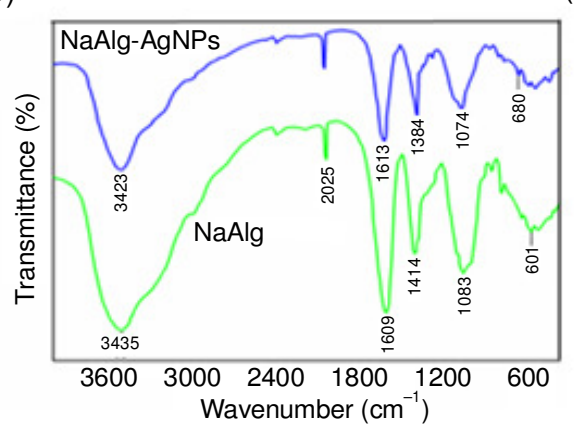

$(\mathrm{F})$

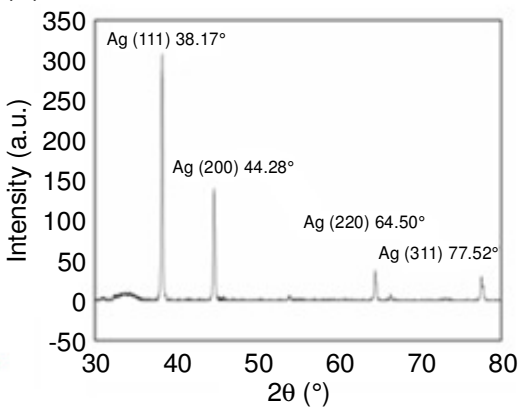

Fig. 1. (A) UV-visible spectra, (B) FT-IR spectra, (C) SEM, (D) TEM, (E) EDS and (F) XRD of AgNPs 
due to crystalline and amorphous natural stages, accompanying crystallized AgNPs. In addition to the Bragg peaks adumbrative of silver nanocrystals, added peaks were as well observed, although they were not assigned to the spectrum and may accept been due to amoebic compounds, responsible for silver ion reduction and the stabilization of resultant nanoparticles [29].

Optimization of treatment conditions: Fig. 2a represents the effect of $\mathrm{pH}$ on colour strength $(\mathrm{K} / \mathrm{S})$ values of wool treated by 35 and 70 ppm AgNPs with respect to controlled conditions. When the $\mathrm{pH}$ of AgNPs solution was 2, the solution was almost colourless after treatment of wool fabrics and the assembling trend of AgNPs was the same as that at $\mathrm{pH} 4$. But still better result observed when $\mathrm{pH}$ is 4 rather than $\mathrm{pH} 2$ in terms of $\mathrm{K} / \mathrm{S}$ value. From Fig. 2a, it can be depicted that maximum colour strength (K/S) value obtained at $\mathrm{pH} 4$, a significant proportion of internal amino groups are protonated, leading to a neutralization of this surface charge. However, the carboxylate anions are not substantially protonated until the $\mathrm{pH}$ approaches high acidic medium. This is the reason for the use of acid when dyeing wool with highly hydrophilic leveling acid dyes and it is clear from Fig. 2a that the AgNPs behave in a similar manner to those dyes [30]. However, AgNPs deposition did not occur when the application medium was approximately neutral condition like $\mathrm{pH} 7$ as there is no significant change in K/S value. Similar phenomenon has been observed when the solution was in highly alkaline medium like $\mathrm{pH} 11$. In addition, $\mathrm{pH}$ value played a vital contribution in the AgNPs treatment process because of the different surface characteristics of wool polymer. So, it could be concluded that AgNPs on wool surface is applicable may be due to the electrostatic interaction between carboxylate groups of AgNPs and amino groups of wool polymer. Optimization of treatment time can be an important step in sustainable textile functionalization with respect to the energy savings. Fig. $2 b$ shows the progress of wool treatments with different concentration of AgNPs at times ranging from $30 \mathrm{~min}$ to $4 \mathrm{~h}$ in terms of K/S value. The results revealed that $\mathrm{K} / \mathrm{S}$ value increases from 0.5 to $2 \mathrm{~h}$ time interval. This can be attributed due to the changing pattern of wool materials and the zeta potential of AgNPs. Because the wool surface having positive charge and AgNPs surface having negative charge resulting a strong attraction between them [31]. Therefore, up to $2 \mathrm{~h}$ of exhaustion time, the rate of AgNPs uptake by wool was very high; however, there was no significant change in curve but asymptotic behaviour was observed beyond $2 \mathrm{~h}$. The application of AgNPs on wool fabrics can be controlled by the temperature of application medium. Fig. 2c evident that the rate of AgNPs uptake by wool samples were increased up to $40{ }^{\circ} \mathrm{C}$ and thus completed in $2 \mathrm{~h}$. An interesting correlation between temperature and time was also observed in this experiment. When the temperature was increased to $85^{\circ} \mathrm{C}$, the deposition was finished in $1 \mathrm{~h}$ at same concentration because of higher kinetic energy of the system at higher temperature [32].

FT-IR: Fig. 3 shows the FT-IR spectra of untreated wool and AgNPs treated wool fabrics. Wool is composed of a cuticle and cortex, whereas the cortex makes up the main portion of the wool. It is made up of more than 18 amino acids, which can be alienated into four distinct groups: cationic, anionic, non-polar and polar. The main functional groups include carboxyl, amino and hydroxyl groups [33]. Overall, all wool fibers exhibited similar absorption at $3260 \mathrm{~cm}^{-1}(\mathrm{~N}-\mathrm{H}$ and $\mathrm{O}-\mathrm{H}), 2881 \mathrm{~cm}^{-1}$ $\left(-\mathrm{CH}_{2}\right), 1670 \mathrm{~cm}^{-1}$ (amide I), $1540 \mathrm{~cm}^{-1}$ (amide II) and 1270
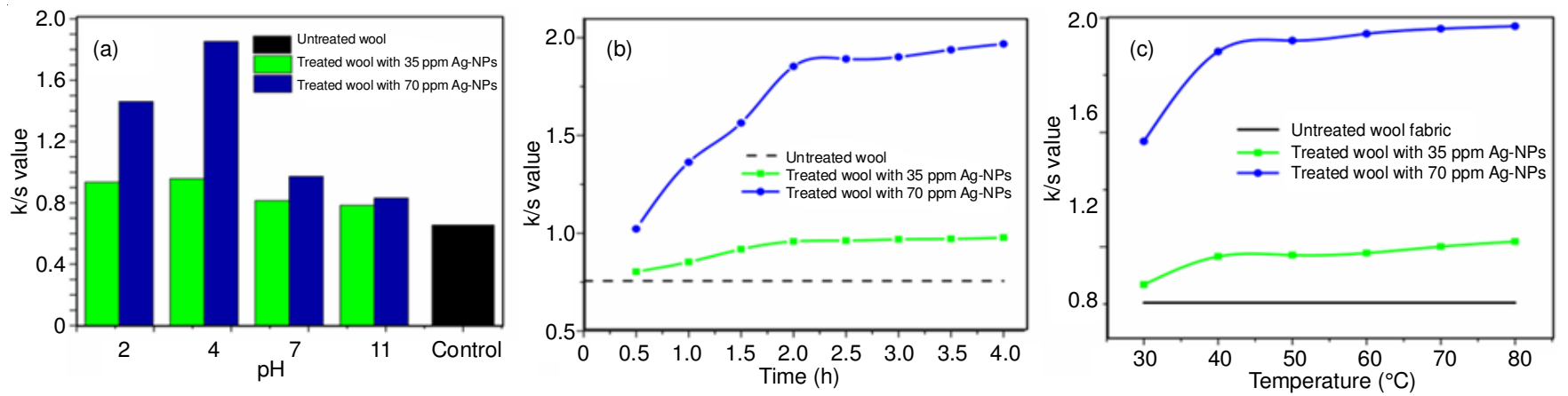

Fig. 2. Effect of the (A) $\mathrm{pH}$ (time $40 \mathrm{~min}$ and temp $40{ }^{\circ} \mathrm{C}$ ), (B) temperature (pH 4 and time $40 \mathrm{~min}$ ) and (C) effect of the application time (pH 4 and temp $40{ }^{\circ} \mathrm{C}$ ) on the particle uptake in terms of $\mathrm{K} / \mathrm{S}$ value
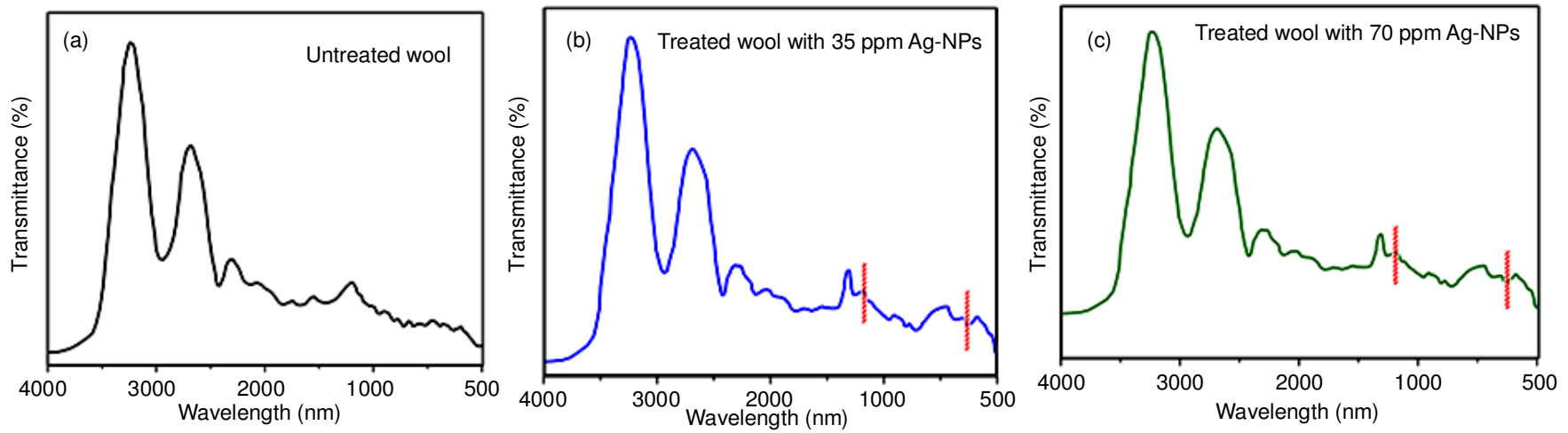

Fig. 3. FTIR spectra of control wool fabric and AgNPs-modified wool fabric 
$\mathrm{cm}^{-1}$ (amide III). When spectra of untreated Fig. $6 \mathrm{a}$ and treated Fig. 4b and 4c were compared, new peaks at 1260 and 1100 $\mathrm{cm}^{-1}$ were observed. The intensity of these peaks is stronger than the peaks for the spectrum of untreated wool, which is due to interaction between $\mathrm{OH}$ of $\mathrm{Na}-\mathrm{Alg}$ and amide bond of the wool. This suggests that AgNPs were successfully adsorbed on wool and confirms the two new peaks in spectra are derived from the interaction of wool polymer system and AgNPs [34].

Thermal stability: Thermal stability of control wool fabric and AgNPs functionalized wool fabric were analyzed with TGA (Fig. 4). The untreated wool fabric after the loss of regains water (occurring from 30 to $160{ }^{\circ} \mathrm{C}$ and is accompanied by a decrease of $7 \%$ in wool fibers mass and is ascribed to the loss of water), the initial thermocracking reaction begins at about $280{ }^{\circ} \mathrm{C}$ and the weight became reduced. This is because of destruction of disulphide linkages and the elimination of $\mathrm{H}_{2} \mathrm{~S}$, followed by the thermal pyrolysis of chain linkages, peptide bridges and some other lateral chains, which finally leads to backbone degradation $[35,36]$. On the other hand, wool fabrics treated with AgNPs were found to have a delayed thermocracking reaction. So, AgNPs treating of wool fabrics lead to increase its thermal stability, which is assured the consequence of AgNPs in advanced treating effectiveness. This is because $\mathrm{Na}-\mathrm{Alg}$ and silver nanocomposite may act as cross linker or filler of wool polymer system; therefore, heat has significant effect on the treated fabrics. Thus it is concluded that the untreated wool have a higher weight loss, while the highest AgNPs concentration offered the lowest weight loss.

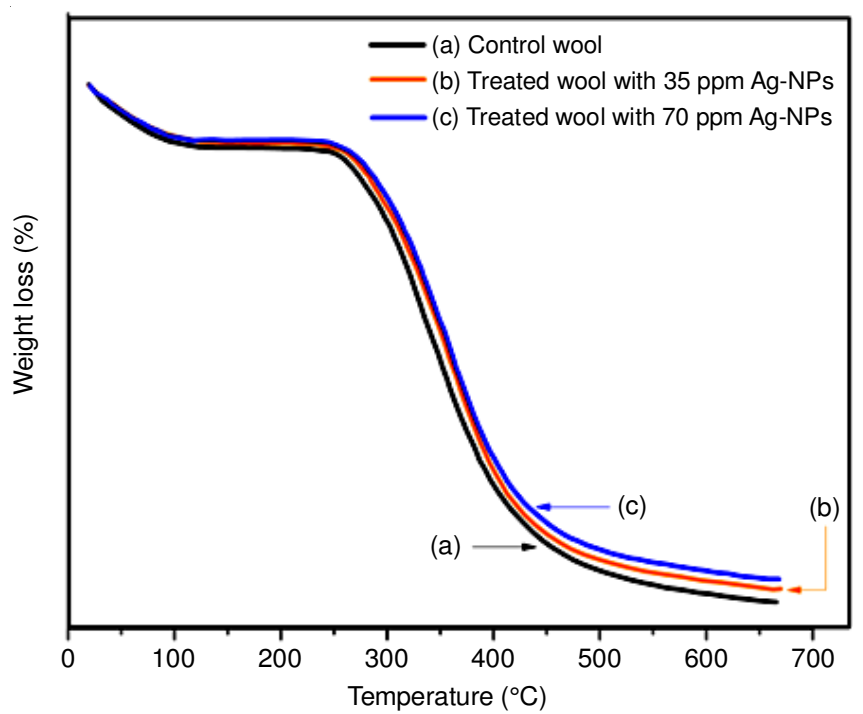

Fig. 4. TG curves of the AgNPs loaded wool

UV protection: The actual damage to human skin from $\mathrm{UV}$ radiation is in the range of UV-A (320-400 nm). Recently, metal nanoparticles and metal salts have been used as UV protecting agent [37]. Therefore, the effect of green based silver nanoparticles on the optical properties of fabrics, the UV transmittance spectroscopy of untreated and treated fabrics was measured at the wavelength range of $200-440 \mathrm{~nm}$ (Fig. 5). The transmittance spectra demonstrated an obvious distinction among all samples. Typically, the untreated wool fabric showed the highest UV transmittance percentage compared to those treated, which supports that wool fabric had no significant effect on UV protection properties. Once wool fabric was functionalized with 35 ppm AgNPs, the corresponding spectrum of wool-AgNPs decreased to about $46 \%$ in comparison with the raw wool fabric. It was found that the incorporation of $70 \mathrm{ppm}$ AgNPs on fabric-treated clearly reduces UV transmittance to $84 \%$, suggesting that AgNPs possess high protection from UV rays. These results indicated the ability of AgNPs treated fabrics to block UV rays. The high UV protection of AgNPs incorporated finished fabrics could be due to the large refractive index of AgNPs resulting in very efficient UV scattering [38]. Previous studies [39] have revealed that the AgNPs enhances the UV protection property of fabric due to the plasmon structure of AgNPs. Therefore, present results indicated that green based AgNPs absorb the UV rays effectively.

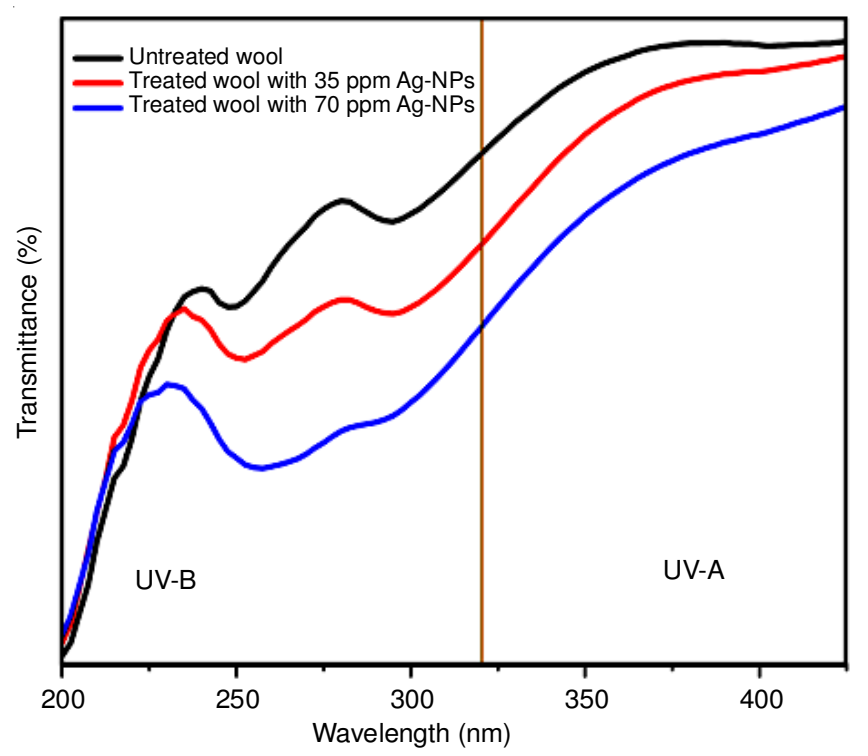

Fig. 5. UV transmittance rate of fabrics

Antibacterial properties: To examine the bacterial inhibition efficacy of AgNPs treated fabrics zone of inhibition assay i.e., qualitative analysis has been applied with agar diffusion test method. Fabric samples were placed on bacteria-inoculated agar plates and were visualized for antibacterial activity (Fig. 6 ). The performance of bacterial growth was observed on fabric surface that treated with commercial antibacterial agent and AgNPs agent. Table-1 presented the inhibitory effect of green AgNPs with different diameters of clear zone of inhibition around the fabric swatches.

TABLE-1

ANTIBACTERIAL ACTIVITY OF WOOL FABRIC COATED WITH GREEN BASED AgNPs

\begin{tabular}{lcc|cc}
\hline \multirow{2}{*}{ Samples } & \multicolumn{2}{c|}{ Bacterial reduction $(\%)$} & \multicolumn{2}{c}{ Zone of inhibition $(\mathrm{mm})$} \\
\cline { 2 - 5 } & E. coli & S. aureus & E. coli & S. aureus \\
\hline Commercial & $94 \pm 2.21$ & $96 \pm 4.21$ & $27 \pm 1.02$ & $28 \pm 0.54$ \\
35 ppm AgNPs & $89 \pm 1.12$ & $90 \pm 2.12$ & $22 \pm 0.43$ & $25 \pm 0.68$ \\
70 ppm AgNPs & $91 \pm 2.06$ & $93 \pm 5.06$ & $23 \pm 0.4$ & $26 \pm 0.48$ \\
\hline
\end{tabular}

The inhibition zone test results (Fig. 6) clearly show that commercial antibacterial agent display highest inhibition zones for $E$. coli $(27 \pm 1.02)$ and $S$. aureus $(28 \pm 0.54)$. In contrast, 

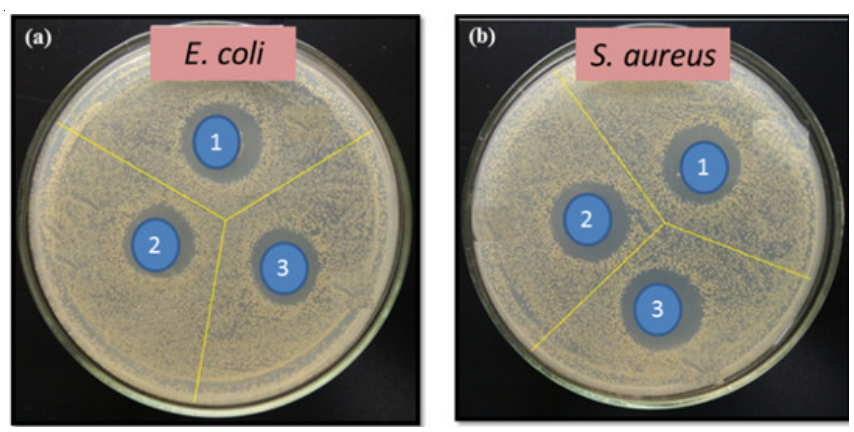

Fig. 6. Antibacterial activity of the commercial (1) and AgNPs-treated wool fabrics $35 \mathrm{ppm}$ (2), $70 \mathrm{ppm}$ (3) on E. coli (a) and S. aureus (b)

AgNPs treated showed inhibition zones around the specimen and the diameter of each transparent zone was close to commercial sample. The difference in the zone of inhibition was likely influenced by the concentrations of AgNPs during the preparation of nanoparticles. As shown in Table-1, the amount of silver ion was higher when the fabric was functionalized using 70 ppm AgNPs which presented a better inhibitory effect against both the microorganisms with a zone of inhibition upto $23 \pm 0.4 \mathrm{~mm}$ in diameter for $E$. coli and $26 \pm 0.48 \mathrm{~mm}$ in diameter for $S$. aureus on the finished wool fabric, where using $35 \mathrm{ppm}$ AgNPs zone of inhibition upto $22 \pm 0.43 \mathrm{~mm}$ in diameter for $E$. coli and $25 \pm 0.68 \mathrm{~mm}$ in diameter for $S$. aureus observed. This phenomenon was attributed to the mechanism of biocidal action of fabric is due to the leached $\mathrm{Ag}^{+}$ions [40]. In addition, AgNPs are extremely reactive with proteins because it adversely affects cellular metabolism and strongly inhibits the cell membrane when AgNPs come in contact with bacteria and fungus. It also suppresses respiration, basal metabolism of the electron transfer system and transport of substrate into the microbial cell membrane and as a result it inhabited the multiplication and expansion of those bacteria and fungi which cause infection, odour, itchiness and sores.

The quantitative assessment of antibacterial activity of functionalized wool fabric with synthesized green based AgNPs was performed according to the percent reduction method (AATCC 100). Silver nanoparticles have a significant influence on bacterial reduction; in case of both concentrations of AgNPs induced the inhibition rate to increase to above $90 \%$. When the concentration of AgNPs increased, the antibacterial activity against $E$. coli and $S$. aureus improved slowly and stably (Table$1)$. The reason can be defined in terms of a definite degree of sterilizing effect created by the fact of metallic ions as well as metallic compounds. It is perhaps a segment of atmospheric oxygen in water or air is adjusted into active oxygen due to catalysis of metallic ion, thereby dissolving the organic substance to enhance a sterilizing action [41]. Therefore, AgNPs are able to increase their contact with microbes because of their extremely large relative surface area, results in enhancement of antimicrobial activity. The antibacterial performance demonstrated in above experiments is perfectly in agreement with the broad spectrum of antimicrobial activity of AgNPs and it could be a good competitor for conventional antibacterial agents.

\section{Conclusion}

In conclusion, an easy and efficient approach is reported to obtain wool fabric with durable UV resistance and antibac- terial activity by incorporating green based AgNPs finishing. Two different concentrations were used for preparation of AgNPs using green method. Intensive investigation into the characterization of the treated fabrics along with thermal properties of AgNPs treated wool was carried out. The treated fabrics were showed excellent bacterial reduction with values exceed $90 \%$ and imparted an excellent UV protection, particularly 70 ppm AgNPs concentration on wool fabric performed better in comparison with 35 ppm AgNPs. Finally, it was anticipated that the output of present research will be promising in terms of environmental friendly functionalization on wool for the advancement of its regular properties through well-synthesized green AgNPs.

\section{ACKNOWLEDGEMENTS}

The authors thank Runhe Chemical Industry, China and Color Root (Hubei) Technology Limited, China, for providing the technical support and the School of Chemistry \& Chemical Engineering, Wuhan Textile University, China, for providing necessary research facilities.

\section{REFERENCES}

1. H. Xie, S. Li and S. Zhang, Green Chem., 7, 606 (2005); https://doi.org/10.1039/b502547h.

2. S.-S. Sun and R.-C. Tang, Ind. Eng. Chem. Res., 50, 4217 (2011); https://doi.org/10.1021/ie101505q.

3. M. Montazer and S. Seifollahzadeh, Photochem. Photobiol., 87, 877 (2011);

https://doi.org/10.1111/j.1751-1097.2011.00917.x.

4. R. Dastjerdi and M. Montazer, Colloids Surf. B Biointerfaces, 79, 5 (2010); https://doi.org/10.1016/j.colsurfb.2010.03.029.

5. L. Hadad, N. Perkas, Y. Gofer, J. CalderonMoreno, A. Ghule and A. Gedanken, J. Appl. Polym. Sci., 104, 1732 (2007); https://doi.org/10.1002/app.25813.

6. D. Zhang, G.W. Toh, H. Lin and Y. Chen, J. Mater. Sci., 47, 5721 (2012); https://doi.org/10.1007/s10853-012-6462-7.

7. W. Chen, J. Yan, N. Song, Q. Li, B. Yang and Y. Dai, Guijinshu, 27, 14 (2006).

8. N. Choi, D. Seo and J. Lee, Mater. Sci. Forum, 29, 394 (2005).

9. J. Yan, H. Tao, M. Zeng, J. Tao, S. Zhang, Z. Yan, W. Wang and J. Wang, Chin. J. Catal., 30, 856 (2009); https://doi.org/10.1016/S1872-2067(08)60128-X.

10. P. Setua, A. Chakraborty, D. Seth, M.U. Bhatta, P. Satyam and N. Sarkar, J. Phys. Chem. C, 111, 3901 (2007); https://doi.org/10.1021/jp067475i.

11. J.J.A. Bonilla, D.J.P. Guerrero, R.G.T. Sáez, K. Ishida, B.B. Fonseca, S. Rozental and C.C.O. López, J. Nanosci. Nanotechnol., 17, 1729 (2017); https://doi.org/10.1166/jnn.2017.12822.

12. D. Wei, W. Sun, W. Qian, Y. Ye and X. Ma, Carbohydr. Res., 344, 2375 (2009); https://doi.org/10.1016/j.carres.2009.09.001.

13. Y. Park, Y. Hong, A. Weyers, Y. Kim and R. Linhardt, IET Nanobiotechnol., 5, 69 (2011); https://doi.org/10.1049/iet-nbt.2010.0033.

14. J.A. Maclaren and B. Milligan, Wool Science: The Chemical Reactivity of the Wool Fibre, Science Press: Marrickville, Australia, vol. 122. (1981).

15. M. Pollini, F. Paladini, A. Licciulli, A. Maffezzoli, L. Nicolais and A. Sannino, J. Appl. Polym. Sci., 125, 2239 (2012); https://doi.org/10.1002/app.36444.

16. P.S. Rad, M. Montazer and M.K. Rahimi, J. Appl. Polym. Sci., 122, 1405 (2011); https://doi.org/10.1002/app.34089.

17. H.Y. Ki, J.H. Kim, S.C. Kwon and S.H. Jeong, J. Mater. Sci., 42, 8020 (2007); https://doi.org/10.1007/s10853-007-1572-3.

18. M.N. Pervez, M.A. Rahman, L. Yu and Y. Cai, in MATEC Web of Conferences, vol. 108, 03004 (2017); https://doi.org/10.1051/matecconf/201710803004 
19. A. Slistan-Grijalva, R. Herrera-Urbina, J. Rivas-Silva, M. Ávalos-Borja, F. Castillón-Barraza and A. Posada-Amarillas, Physica E, 27, 104 (2005);

https://doi.org/10.1016/j.physe.2004.10.014

20. K.G. Stamplecoskie and J.C. Scaiano, J. Am. Chem. Soc., 132, 1825 (2010); https://doi.org/10.1021/ja910010b.

21. E. Abdel-Halim and S.S. Al-Deyab, Carbohydr. Polym., 86, 1615 (2011); https://doi.org/10.1016/j.carbpol.2011.06.072.

22. A. Manuja, S. Kumar, N. Dilbaghi, G. Bhanjana, M. Chopra, H. Kaur, R. Kumar, B.K. Manuja, S.K. Singh and S.C. Yadav, Nanomedicine, 9 , 1625 (2014); https://doi.org/10.2217/nnm.13.148.

23. G. CardenasJiron, D. Leal, B. Matsuhiro and I. Osorio-Roman, J. Raman Spectrosc., 42, 870 (2011); https://doi.org/10.1002/jrs.2760.

24. C. Sartori, D.S. Finch, B. Ralph and K. Gilding, Polymer, 38, 43 (1997); https://doi.org/10.1016/S0032-3861(96)00458-2.

25. A. Mandal, S. Sekar, N. Chandrasekaran, A. Mukherjee and T.P. Sastry, RSC Adv., 5, 15763 (2015) https://doi.org/10.1039/C4RA09694K.

26. P. Devaraj, P. Kumari, C. Aarti and A. Renganathan, J. Nanotechnol. Article ID 598328 (2013); https://doi.org/10.1155/2013/598328.

27. M. El-Rafie, M. El-Naggar, M. Ramadan, M.M. Fouda, S.S. Al-Deyab and A. Hebeish, Carbohydr. Polym., 86, 630 (2011); https://doi.org/10.1016/j.carbpol.2011.04.088.

28. A.M. Fayaz, K. Balaji, M. Girilal, P. Kalaichelvan and R. Venkatesan, J. Agric. Food Chem., 57, 6246 (2009); https://doi.org/10.1021/jf900337h.

29. S.M. Roopan, Rohit, G. Madhumitha, A.A. Rahuman, C. Kamaraj, A. Bharathi and T.V. Surendra, Ind. Crops Prod., 43, 631 (2013); https://doi.org/10.1016/j.indcrop.2012.08.013.
30. T. Briggs and A. Bull, J. Phys. Chem., 26, 845 (1921); https://doi.org/10.1021/j150225a005.

31. B. Tang, J. Wang, S. Xu, T. Afrin, W. Xu, L. Sun and X. Wang, J. Colloid Interface Sci., 356, 513 (2011); https://doi.org/10.1016/j.jcis.2011.01.054

32. D.G. King and A.P. Pierlot, Color. Technol., 125, 111 (2009); https://doi.org/10.1111/j.1478-4408.2009.00182.x.

33. S.H. Hsieh, Z. Huang, Z. Huang and Z. Tseng, J. Appl. Polym. Sci., 94, 1999 (2004); https://doi.org/10.1002/app.21104.

34. P.P. Bose, M.G. Drew and A. Banerjee, Org. Lett., 9, 2489 (2007); https://doi.org/10.1021/ol0708471.

35. P.J. Davies, A.R. Horrocks and M. Miraftab, Polym. Int., 49, 1125 (2000); https://doi.org/10.1002/1097-0126(200010)49:10<1125::AIDPI489>3.0.CO:2-B.

36. M. Forouharshad, M. Montazer, M.B. Moghadam and O. Saligheh, Thermochim. Acta, 516, 29 (2011); https://doi.org/10.1016/j.tca.2011.01.007.

37. H.E. Emam and T. Bechtold, Appl. Surf. Sci., 357, 1878 (2015); https://doi.org/10.1016/j.apsusc.2015.09.095.

38. M. Gorenšek and P. Recelj, Text. Res. J., 77, 138 (2007); https://doi.org/10.1177/0040517507076329.

39. C.-H. Xue, J. Chen, W. Yin, S.-T. Jia and J.-Z. Ma, Appl. Surf. Sci., 258, 2468 (2012) https://doi.org/10.1016/j.apsusc.2011.10.074.

40. T. Maneerung, S. Tokura and R. Rujiravanit, Carbohydr. Polym., 72, 43 (2008); https://doi.org/10.1016/j.carbpol.2007.07.025.

41. M. Saito, J. Coated Fabrics, 23, 150 (1993); https://doi.org/10.1177/152808379302300205. 